the quartz crystal from the normal incidence of the sound beam to light path. These angles of tilt were measured accurately by an optical lever system. We used ethyl iodide as the liquid medium giving sound of wave-length $0.0041 \mathrm{~cm}$. at a frequency of $21 \cdot 05 \mathrm{Mc}$. For the third-order reflexions, however, $n$-hexane was substituted giving sound waves of $0.0051 \mathrm{~cm}$. at the same frequency.

The results for the first, second and third order of the diffraction pattern on one side are given in terms of the Bragg angle in Fig. 1. Here $\alpha / 2$ is the reflexion maximum for the first order.

The first order shows a maximum at $\alpha / 2$ only, while the maxima at $\alpha / 2, \alpha$ and $3 \alpha / 2$ for the second order

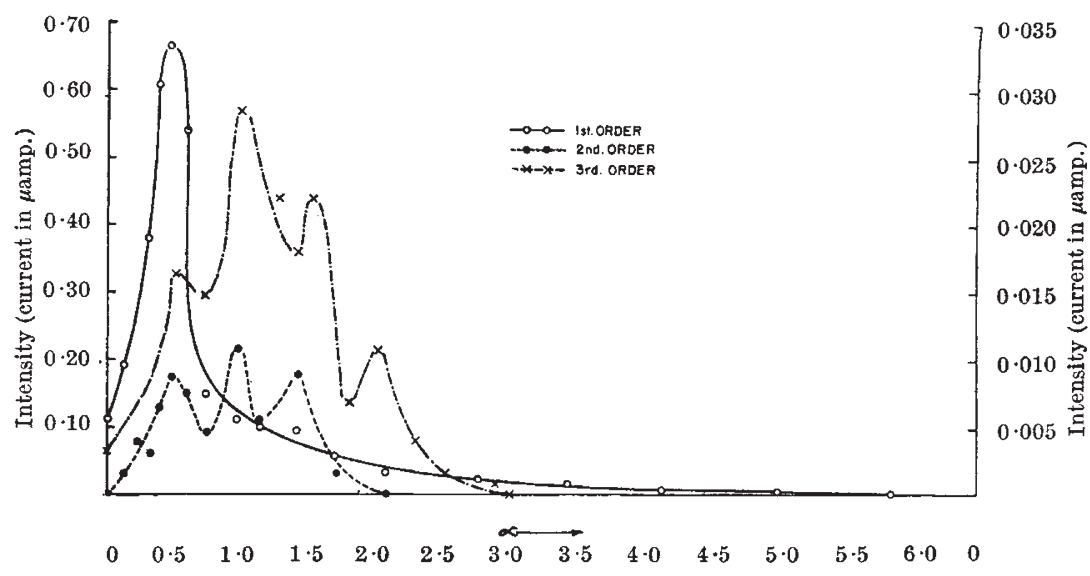

Fig. 1. The abscissa represents the reflexion angle $a$, and the ordinate (left-hand side for the first order and the right-hand side for the second and third orders) represents the intensity expressed in terms of the current in microamperes

The forward reaction can be followed by the changes in absorption spectrum as oxygen is bubbled through the solution of the $\mathrm{Fe}(\mathrm{DMG})_{2}(\mathrm{bas} \theta)_{2}$ complex and the back reaction can be followed by observing similar changes bubbling pure nitrogen through a solution of the oxygenated complex. The spectrophotometric measurements show that the reaction is completely reversible for some of the complexes but that some autoxidation occurs with others. The changes in absorption spectrum with uptake of oxygen are very similar to those which occur on the uptake of carbon monoxide or cyanide by the complex. Both the liberation of oxygen and the liberation of carbon monoxide from the complexes have been studied manometrically. There would appear to be two complexes in one of which two molecules of oxygen or carbon monoxide are bound to the cation and, in the other, one molecule is bound as in the above equation. Comparison of the manometric and spectrophotometric measurements indicates that the first molecule of oxygen or carbon monoxide is picked up slowly but the second molecule is picked up so very slowly that the two stages of the reaction can be followed.

The rate at which oxygen replaces a base molecule in the above equation is sensitive to the base, as is the rate at which the oxygen

are unmistakably clear. $\alpha / 2, \alpha, 3 \alpha / 2$ and $2 \alpha$ are the peaks for the third order line, but perhaps due to the low sound intensity and greater tilt of the quartz crystal the fifth one at $5 \propto / 2$ does not make its appearance markedly. However, these results are in good agreement with Aggarwal's expressions.

It is of interest that a careful study of the photograph published by one of $\mathrm{us}^{4}$ also shows the presence of more than one maximum for the second order.

Full details will be published elsewhere in due course.

$$
\begin{aligned}
& \text { S. Parthasarathy } \\
& \text { C. B. TIPnis }
\end{aligned}
$$

National Physical Laboratory,

New Delhi 12. July 22.

1 Bergmann, L., "Der Ultraschall" (1954). 2 Aggarwal, R. R., Proc. Ind. Acad. Sci., A, 31, 417 (1950); Ph.D.
thesis, University of Delhi (1954).

${ }^{3}$ Aggarwal, R. R., Ph.D. thesis, University of Delhi (1954).

4 Parthasarathy, S., Proc. Ind. Acad. Sci., 3, 594, plate XXXIV (1936).

\section{Uptake of Molecular Oxygen by Ferrous Complexes}

DURING the course of a deliberate study of models for iron porphyrin complexes of biological importance we have shown that ferrous dimethylglyoxime, Fe(DMG), is capable of combining reversibly with molecular oxygen. The reaction occurs in a mixed solvent, 50 per cent aqueous dioxan, in which the complex is appreciably soluble in the presence of various added bases such as pyridine, ammonia, histidine and imidazole. The $p \mathrm{H}$ of the solution must be in the range 7-11 if the reaction is to be observed. We consider the reversible reaction to be $\mathrm{Fe}(\mathrm{DMG})_{2}(\text { base })_{2}+\mathrm{O}_{2} \rightleftharpoons \mathrm{Fe}(\mathrm{DMG})_{2}$ (base) $\mathrm{O}_{2}+$ base molecule can be discharged afterwards. Oxygen is picked up most rapidly by the histidine complex and least rapidly by the pyridine complex. The evolution of oxygen is least rapid in the case of the pyridine complex.

Clearly these complexes parallel the behaviour of different biological complexes some of which pick up oxygen, some of which are autoxidizable and some of which are almost inert to oxygen. We hope to publish a detailed account of our observations together with a correlation between the properties of the models and the properties of the biologically important compounds shortly.

J. F. DRAKE

R. J. P. Williams

Inorganic Chemistry Laboratory,

South Parks Road, Oxford. Sept. 17.

\section{Entropy Production in Irreversible Mechanical Processes}

Is discussing the concept of entropy, many authors relate the 'production of heat' or 'energetic heat evolution' (dQ irrev.) accompanying an irreversible process to the 'loss of useful work' and to the 'irreversible production of entropy' (d S) by an equation of the form ${ }^{1}$ :

$$
\mathrm{d} Q_{\text {irrev. }}=\mathrm{d} W_{\text {irrev. }}-\mathrm{d} W_{\text {rev. }}=T \mathrm{~d}_{i} S
$$

where $\mathrm{d} W_{\text {rev. }}$ and $\mathrm{d} W_{\text {irrev. are, respectively, the }}$ amounts of work received by the system during a reversible and an irreversible change between the same initial and final states of the system; $T$ is the 